\title{
Trace formulae for Schrödinger operators with singular interactions
}

\author{
Jussi Behrndt, Matthias Langer and Vladimir Lotoreichik*
}

\begin{abstract}
Dedicated with great pleasure to our teacher, colleague and friend Pavel Exner on the occasion of his 70th birthday.
\end{abstract}

\begin{abstract}
Let $\Sigma \subset \mathbb{R}^{d}$ be a $C^{\infty}$-smooth closed compact hypersurface, which splits the Euclidean space $\mathbb{R}^{d}$ into two domains $\Omega_{ \pm}$. In this note self-adjoint Schrödinger operators with $\delta$ and $\delta^{\prime}$-interactions supported on $\Sigma$ are studied. For large enough $m \in \mathbb{N}$ the difference of $m$ th powers of resolvents of such a Schrödinger operator and the free Laplacian is known to belong to the trace class. We prove trace formulae, in which the trace of the resolvent power difference in $L^{2}\left(\mathbb{R}^{d}\right)$ is written in terms of Neumann-toDirichlet maps on the boundary space $L^{2}(\Sigma)$.
\end{abstract}

2010 Mathematics Subject Classification. Primary 35P20; Secondary 35J10, 35P25, 47B10, 47F05, 81U99.

Keywords. Trace formula, delta interaction, Schrödinger operator, singular potential.

\section{Introduction}

This paper is strongly inspired by the work of Pavel Exner on Schrödinger operators with singular interactions of $\delta$ and $\delta^{\prime}$-type supported on hypersurfaces in $\mathbb{R}^{d}$. Such operators play an important role in mathematical physics, for instance, in nuclear physics or solid state physics or in connection with photonic crystals or other nanostructures. In the case of a curve in $\mathbb{R}^{2}$ such models are also called "leaky quantum wires". The first rigourous investigations of such operators started in the late 1980 s (see, e.g. $[1,14,15]$ ), and the interest in these operators grew steadily in the last two decades. We refer the reader to the review paper [26], the monograph [30], the references therein and also to the more recent papers $[6,7,10,23,24,29$, $35,44]$.

Let $\Sigma \subset \mathbb{R}^{d}$, where $d \geq 2$, be a $C^{\infty}$-smooth closed compact hypersurface without boundary, which naturally splits the Euclidean space $\mathbb{R}^{d}$ into a bounded domain $\Omega_{-}$and an exterior domain $\Omega_{+}$. Moreover, let $\alpha, \omega \in L^{\infty}(\Sigma)$ be real-valued functions. The Schrödinger operator $\mathrm{H}_{\alpha, \Sigma}$ with $\delta$-interaction of strength $\alpha$ and the Schrödinger operator $\mathrm{K}_{\omega, \Sigma}$ with $\delta^{\prime}$-interaction of strength $\omega$ are formally given by

$$
-\Delta-\alpha \delta(x-\Sigma) \quad \text { and } \quad-\Delta-\omega \delta^{\prime}(x-\Sigma) .
$$

${ }^{*}$ JB gratefully acknowledges financial support by the Austrian Science Fund (FWF): Project P 25162-N26. VL gratefully acknowledges financial support by the Czech Science Foundation (GAČR): Project 14-06818S. 
We define these operators rigorously via quadratic forms; see Definition 1.1 below. Let us first fix some notation. Since the space $L^{2}\left(\mathbb{R}^{d}\right)$ naturally decomposes as $L^{2}\left(\mathbb{R}^{d}\right)=L^{2}\left(\Omega_{+}\right) \oplus L^{2}\left(\Omega_{-}\right)$, we can write functions $u \in L^{2}\left(\mathbb{R}^{d}\right)$ as $u=u_{+} \oplus u_{-}$ with $u_{ \pm}=u \uparrow \Omega_{ \pm} \in L^{2}\left(\Omega_{ \pm}\right)$. The $L^{2}$-based Sobolev spaces of order $s \geq 0$ over $\mathbb{R}^{d}$ and $\Omega_{ \pm}$are denoted by $H^{s}\left(\mathbb{R}^{d}\right)$ and $H^{s}\left(\Omega_{ \pm}\right)$, respectively. Note that the hypersurface $\Sigma$ coincides with the boundaries $\partial \Omega_{ \pm}$of the domains $\Omega_{ \pm}$. Hence, for any $u \in H^{1}\left(\mathbb{R}^{d}\right)$ and $u_{ \pm} \in H^{1}\left(\Omega_{ \pm}\right)$the traces $\left.u\right|_{\Sigma}$ and $\left.u_{ \pm}\right|_{\Sigma}$ on $\Sigma$ are well defined as functions in $L^{2}(\Sigma)$. Further, for a function $u \in H^{1}\left(\mathbb{R}^{d} \backslash \Sigma\right):=H^{1}\left(\Omega_{+}\right) \oplus H^{1}\left(\Omega_{-}\right)$ we define its jump on $\Sigma$ as $[u]_{\Sigma}:=\left.u_{+}\right|_{\Sigma}-\left.u_{-}\right|_{\Sigma}$.

Let us now introduce the following quadratic forms that correspond to the formal expression in (1.1). According to [14, §2], [10, §3.4] and [6, Proposition 3.1], the symmetric quadratic forms

$$
\begin{aligned}
\mathfrak{h}_{\alpha, \Sigma}[u]:=\|\nabla u\|^{2}-\left(\left.\alpha u\right|_{\Sigma},\left.u\right|_{\Sigma}\right)_{\Sigma}, & \operatorname{dom} \mathfrak{h}_{\alpha, \Sigma}:=H^{1}\left(\mathbb{R}^{d}\right), \\
\mathfrak{k}_{\omega, \Sigma}[u]:=\left\|\nabla u_{+}\right\|_{+}^{2}+\left\|\nabla u_{-}\right\|_{-}^{2}-\left(\omega[u]_{\Sigma},[u]_{\Sigma}\right)_{\Sigma}, & \operatorname{dom} \mathfrak{k}_{\omega, \Sigma}:=H^{1}\left(\mathbb{R}^{d} \backslash \Sigma\right),
\end{aligned}
$$

in $L^{2}\left(\mathbb{R}^{d}\right)$ are closed, densely defined and bounded from below; here $u=u_{+} \oplus u_{-}$ with $u_{ \pm}$as above, and $\|\cdot\|_{ \pm}$denotes the norm on $L^{2}\left(\Omega_{ \pm} ; \mathbb{C}^{d}\right)$.

Definition 1.1. Let $\mathrm{H}_{\alpha, \Sigma}$ and $\mathrm{K}_{\omega, \Sigma}$ be the self-adjoint operators in $L^{2}\left(\mathbb{R}^{d}\right)$ corresponding to the forms $\mathfrak{h}_{\alpha, \Sigma}$ and $\mathfrak{k}_{\omega, \Sigma}$, respectively, via the first representation theorem $\left(\left[37\right.\right.$, Theorem VI.2.1]). Moreover, set $\mathrm{H}_{\text {free }}:=\mathrm{H}_{0, \Sigma}(\alpha \equiv 0)$ and $\mathrm{K}_{\mathrm{N}}:=\mathrm{K}_{0, \Sigma}$ $(\omega \equiv 0)$.

The operator $\mathrm{H}_{\alpha, \Sigma}$ is called Schrödinger operator with $\delta$-interaction of strength $\alpha$ supported on $\Sigma$; the operator $\mathrm{K}_{\omega, \Sigma}$ is called Schrödinger operator with $\delta^{\prime}-$ interaction of strength ${ }^{1} \omega$ supported on $\Sigma$. The operator $\mathrm{H}_{\text {free }}$ is the usual free Laplacian on $\mathbb{R}^{d}$, and $\mathrm{K}_{\mathrm{N}}$ is the orthogonal sum of the standard Neumann Laplacians on $\Omega_{+}$and $\Omega_{-}$. Let us mention that the operators $\mathrm{H}_{\alpha, \Sigma}$ and $\mathrm{K}_{\omega, \Sigma}$ can also be introduced via interface conditions at the hypersurface $\Sigma$; see, e.g. [10].

The aim of this paper is to derive trace formulae for $\mathrm{H}_{\alpha, \Sigma}$ and $\mathrm{K}_{\omega, \Sigma}$. According to [10] for $m \in \mathbb{N}$ the resolvent power differences

$$
\begin{array}{ll}
\left(\mathrm{H}_{\alpha, \Sigma}-\lambda\right)^{-m}-\left(\mathrm{H}_{\text {free }}-\lambda\right)^{-m}, & m>\frac{d-2}{2}, \quad \lambda \in \rho\left(\mathrm{H}_{\alpha, \Sigma}\right), \\
\left(\mathrm{K}_{\omega, \Sigma}-\lambda\right)^{-m}-\left(\mathrm{H}_{\text {free }}-\lambda\right)^{-m}, & m>\frac{d-1}{2}, \quad \lambda \in \rho\left(\mathrm{K}_{\omega, \Sigma}\right),
\end{array}
$$

are in the trace class. Their traces as functions of $\lambda$ or as functions of interaction strengths are expected to encode a lot of information on the operators $\mathrm{H}_{\alpha, \Sigma}$ and $\mathrm{K}_{\omega, \Sigma}$ themselves and on the shape of $\Sigma$. Such non-trivial connections have been observed in various other settings in the classical papers [18, 36, 42] and more recently in, e.g. [5, 34, 38, 39, 46].

The main results of the paper (see Theorems 1.2 and 1.3) are formulae that express the traces of the resolvent power differences in (1.2) in terms of traces

\footnotetext{
${ }^{1}$ We point out that, in the case of invertible $\omega$, not $\omega$ itself, but its inverse is frequently called the strength of the $\delta^{\prime}$-interaction.
} 
of derivatives of certain operator-valued functions in the boundary space $L^{2}(\Sigma)$. These operator-valued functions are, in turn, expressed in terms of Neumann-toDirichlet maps on $\Omega_{ \pm}$corresponding to the differential expression $-\Delta-\lambda$ and in terms of the coupling functions $\alpha, \omega$. Trace formulae of this kind are useful (see, e.g. $[19,20,32])$ in connection with the estimation of the spectral shift function.

1.1. Traces, Neumann-to-Dirichlet maps and some operator functions. We first recall some notions that are needed in order to formulate the main results of this paper. For a compact operator $K$ in a Hilbert space $\mathcal{H}$ we define its singular values $s_{k}(K), k=1,2, \ldots$, as the eigenvalues of the non-negative compact operator $|K|=\left(K^{*} K\right)^{1 / 2} \geq 0$ in $\mathcal{H}$ ordered in non-decreasing way and with multiplicities taken into account. If $\sum_{k=1}^{\infty} s_{k}(K)<\infty$, we say that $K$ belongs to the trace class and define its trace as

$$
\operatorname{Tr} K:=\sum_{k=1}^{\infty} \lambda_{k}(K),
$$

where $\lambda_{k}(K)$ are the eigenvalues of $K$ repeated with their algebraic multiplicities. Note also that the series in the definition of the trace converges absolutely.

Let us also define some auxiliary maps associated with partial differential equations. For the sake of brevity, we introduce the spaces

$$
H_{\Delta}^{3 / 2}\left(\Omega_{ \pm}\right):=\left\{u_{ \pm} \in H^{3 / 2}\left(\Omega_{ \pm}\right): \Delta u_{ \pm} \in L^{2}\left(\Omega_{ \pm}\right)\right\}
$$

For any $u_{ \pm} \in H_{\Delta}^{3 / 2}\left(\Omega_{ \pm}\right)$its Neumann trace $\left.\partial_{\nu_{ \pm}} u_{ \pm}\right|_{\Sigma}$ exists as a function in $L^{2}(\Sigma)$; see, e.g. $[43, \S 2.7 .3]$. For every $\lambda \in \mathbb{C} \backslash \mathbb{R}_{+}$(where $\mathbb{R}_{+}:=[0, \infty)$ ) and every $\varphi \in L^{2}(\Sigma)$ the boundary value problems

$$
\begin{aligned}
-\Delta u_{ \pm}=\lambda u_{ \pm} & \text {in } \Omega_{ \pm}, \\
\left.\partial_{\nu_{ \pm}} u_{ \pm}\right|_{\Sigma}=\varphi & \text { on } \Sigma,
\end{aligned}
$$

have unique solutions $u_{\lambda, \pm}(\varphi) \in H_{\Delta}^{3 / 2}\left(\Omega_{ \pm}\right)$; see, e.g. [43, §2.7.3]. The operatorvalued functions $\lambda \mapsto M_{ \pm}(\lambda), \lambda \in \mathbb{C} \backslash \mathbb{R}_{+}$, are then defined as

$$
M_{ \pm}(\lambda): L^{2}(\Sigma) \rightarrow L^{2}(\Sigma), \quad M_{ \pm}(\lambda) \varphi:=\left.u_{\lambda, \pm}(\varphi)\right|_{\Sigma}
$$

For fixed $\lambda \in \mathbb{C} \backslash \mathbb{R}_{+}$the operators $M_{ \pm}(\lambda)$ are the Neumann-to-Dirichlet maps for the differential expression $-\Delta-\lambda$ on the domains $\Omega_{ \pm}$. The operators $M_{ \pm}(\lambda)$ are compact and injective and their inverses are called Dirichlet-to-Neumann maps. Recently, there has been a considerable growth of interest in the investigation of these maps (see, e.g. $[3,4,25]$ ), in particular also with the aim to derive spectral properties of the corresponding partial differential operators (see, e.g. [2, 13, 31]).

Further, we define the following operator-valued functions $\lambda \mapsto \widetilde{M}(\lambda), \widehat{M}(\lambda)$, $\lambda \in \mathbb{C} \backslash \mathbb{R}_{+}$, by

$$
\widetilde{M}(\lambda):=\left(M_{+}(\lambda)^{-1}+M_{-}(\lambda)^{-1}\right)^{-1}, \quad \widehat{M}(\lambda):=M_{+}(\lambda)+M_{-}(\lambda) .
$$


We should mention that for every $\lambda \in \mathbb{C} \backslash \mathbb{R}_{+}$the operator $M_{+}(\lambda)^{-1}+M_{-}(\lambda)^{-1}$ is invertible and therefore $\widetilde{M}(\lambda)$ is well defined. Moreover, $\widetilde{M}(\lambda)$ and $\widehat{M}(\lambda)$ are compact operators in $L^{2}(\Sigma)$ for every $\lambda \in \mathbb{C} \backslash \mathbb{R}_{+}$; see [10, Propositions 3.2 and 3.8]. It is worth mentioning that $\widetilde{M}(\lambda)$ and the inverse of $\widehat{M}(\lambda)$ appear naturally in the theory of boundary integral operators. They are used in the treatment of partial differential equations from both analytical [45] and computational [48] viewpoints. The operator-valued function $\widetilde{M}(\cdot)$ was successfully applied to the spectral analysis of the operator $\mathrm{H}_{\alpha, \Sigma}$ in quite a few papers; see, e.g. [27, 28, 30,41] and the survey paper [26].

1.2. Statement of the main results. In the first main result of this note we obtain a trace formula for the resolvent power difference of the operators $\mathrm{H}_{\alpha, \Sigma}$ and $\mathrm{H}_{\text {free. }}$.

Theorem 1.2. Let the self-adjoint operators $\mathrm{H}_{\text {free }}$ and $\mathrm{H}_{\alpha, \Sigma}$ with $\alpha \in L^{\infty}(\Sigma ; \mathbb{R})$ be as in Definition 1.1, and let the operator-valued function $\widetilde{M}$ be as in (1.4). Then for all $m \in \mathbb{N}$ such that $m>\frac{d-2}{2}$ and all $\lambda \in \rho\left(\mathrm{H}_{\alpha, \Sigma}\right)$ the resolvent power difference

$$
\widetilde{D}_{\alpha, m}(\lambda):=\left(\mathrm{H}_{\alpha, \Sigma}-\lambda\right)^{-m}-\left(\mathrm{H}_{\text {free }}-\lambda\right)^{-m}
$$

belongs to the trace class, and its trace can be expressed as

$$
\operatorname{Tr}\left(\widetilde{D}_{\alpha, m}(\lambda)\right)=\frac{1}{(m-1) !} \operatorname{Tr}\left(\frac{\mathrm{d}^{m-1}}{\mathrm{~d} \lambda^{m-1}}\left((I-\alpha \widetilde{M}(\lambda))^{-1} \alpha \widetilde{M}^{\prime}(\lambda)\right)\right) .
$$

In the second main result of this note we obtain trace formulae for the resolvent power differences of the pairs of operators $\left\{\mathrm{K}_{\omega, \Sigma}, \mathrm{K}_{\mathrm{N}}\right\}$ and $\left\{\mathrm{K}_{\omega, \Sigma}, \mathrm{H}_{\text {free }}\right\}$.

Theorem 1.3. Let the self-adjoint operators $\mathrm{H}_{\text {free }}, \mathrm{K}_{\mathrm{N}}$ and $\mathrm{K}_{\omega, \Sigma}$ with $\omega \in L^{\infty}(\Sigma ; \mathbb{R})$ be as in Definition 1.1, and let the operator-valued function $\widehat{M}$ be as in (1.4). Then the following statements hold.

(i) For all $m \in \mathbb{N}$ such that $m>\frac{d-2}{2}$ and all $\lambda \in \rho\left(\mathrm{K}_{\omega, \Sigma}\right)$ the resolvent power difference

$$
\widehat{E}_{\omega, m}(\lambda):=\left(\mathrm{K}_{\omega, \Sigma}-\lambda\right)^{-m}-\left(\mathrm{K}_{\mathrm{N}}-\lambda\right)^{-m}
$$

belongs to the trace class, and its trace can be expressed as

$$
\operatorname{Tr}\left(\widehat{E}_{\omega, m}(\lambda)\right)=\frac{1}{(m-1) !} \operatorname{Tr}\left(\frac{\mathrm{d}^{m-1}}{\mathrm{~d} \lambda^{m-1}}\left((I-\omega \widehat{M}(\lambda))^{-1} \omega \widehat{M}^{\prime}(\lambda)\right)\right) .
$$

(ii) For all $m \in \mathbb{N}$ such that $m>\frac{d-1}{2}$ and all $\lambda \in \rho\left(\mathrm{K}_{\omega, \Sigma}\right)$ the resolvent power difference

$$
\widehat{D}_{\omega, m}(\lambda):=\left(\mathrm{K}_{\omega, \Sigma}-\lambda\right)^{-m}-\left(\mathrm{H}_{\text {free }}-\lambda\right)^{-m}
$$

belongs to the trace class, and its trace can be expressed as

$$
\operatorname{Tr}\left(\widehat{D}_{\omega, m}(\lambda)\right)=\frac{1}{(m-1) !} \operatorname{Tr}\left(\frac{\mathrm{d}^{m-1}}{\mathrm{~d} \lambda^{m-1}}\left((I-\omega \widehat{M}(\lambda))^{-1} \widehat{M}(\lambda)^{-1} \widehat{M}^{\prime}(\lambda)\right)\right) .
$$


We remark that it is also implicitly shown that the derivatives of the operatorvalued functions appearing in the trace formulae exist in a suitable sense and that these derivatives belong to the trace class.

The main ingredients used in the proofs, which are given in Section 3, are Krein-type resolvent formulae, properties of weak Schatten-von Neumann classes, asymptotics of eigenvalues of the Laplace-Beltrami operator on $\Sigma$, and elements of elliptic regularity theory. We point out that for the proof of Theorem 1.3 (ii) an auxiliary trace formula for the resolvent power differences of $\mathrm{H}_{\text {free }}$ and $\mathrm{K}_{\mathrm{N}}$ is derived in Lemma 3.4. This trace formula is also of certain independent interest. We also mention that a similar strategy of proof was employed in our previous paper [12] where we proved trace formulae for generalized Robin Laplacians.

\section{Preliminaries}

This section consists of five subsections. In Subsection 2.1 we recall the notion of weak Schatten-von Neumann classes and their connection with the trace class, and in Subsection 2.2 we collect certain formulae that involve derivatives of holomorphic operator-valued functions. Next, in Subsection 2.3 we recall the definitions of quasi boundary triples and associated $\gamma$-fields and Weyl functions. Krein's resolvent formulae and sufficient conditions for self-adjointness of extensions are discussed in Subsection 2.4. Finally, in Subsection 2.5 we introduce specific quasi boundary triples, which are used to parameterize Schrödinger operators with singular interactions from Definition 1.1.

2.1. $\mathfrak{S}_{p, \infty}$-classes and the trace mapping. Let $\mathcal{H}$ and $\mathcal{K}$ be Hilbert spaces. Denote by $\mathfrak{S}_{\infty}(\mathcal{H}, \mathcal{K})$ the class of all compact operators $K: \mathcal{H} \rightarrow \mathcal{K}$. Recall that, for $p>0$, the weak Schatten-von Neumann ideal $\mathfrak{S}_{p, \infty}(\mathcal{H}, \mathcal{K})$ is defined by

$$
\mathfrak{S}_{p, \infty}(\mathcal{H}, \mathcal{K}):=\left\{K \in \mathfrak{S}_{\infty}(\mathcal{H}, \mathcal{K}): s_{k}(K)=\mathrm{O}\left(k^{-1 / p}\right), k \rightarrow \infty\right\} .
$$

Often we just write $\mathfrak{S}_{p, \infty}$ instead of $\mathfrak{S}_{p, \infty}(\mathcal{H}, \mathcal{K})$. For $0<p^{\prime}<p$ the inclusion

$$
\mathfrak{S}_{p, \infty} \subset \mathfrak{S}_{p^{\prime}, \infty}
$$

holds, and for $s, t>0$ one has

$$
\mathfrak{S}_{\frac{1}{s}, \infty} \cdot \mathfrak{S}_{\frac{1}{t}, \infty}=\mathfrak{S}_{\frac{1}{s+t}, \infty}
$$

where a product of operator ideals is defined as the set of all products. We refer the reader to [33, §§III.7 and III.14] and [47, Chapter 2] for a detailed study of the classes $\mathfrak{S}_{p, \infty}$; see also [11, Lemma 2.3]. If $K \in \mathfrak{S}_{p, \infty}$ with $p<1$, then $K$ belongs to the trace class. It is well known (see, e.g. [33, §III.8]) that, for trace class operators $K_{1}, K_{2}$, the operator $K_{1}+K_{2}$ is also in the trace class, and that

$$
\operatorname{Tr}\left(K_{1}+K_{2}\right)=\operatorname{Tr} K_{1}+\operatorname{Tr} K_{2}
$$


Moreover, if $K_{1} \in \mathcal{B}(\mathcal{H}, \mathcal{K})$ and $K_{2} \in \mathcal{B}(\mathcal{K}, \mathcal{H})$ are such that both products $K_{1} K_{2}$ and $K_{2} K_{1}$ are in the trace class, then

$$
\operatorname{Tr}\left(K_{1} K_{2}\right)=\operatorname{Tr}\left(K_{2} K_{1}\right) .
$$

The next useful lemma is a special case of [11, Lemma 4.7] and is based on the asymptotics of the eigenvalues of the Laplace-Beltrami operator. For a smooth compact manifold $\Sigma$ we denote the usual $L^{2}$-based Sobolev spaces by $H^{r}(\Sigma), r \geq 0$.

Lemma 2.1. Let $\Sigma$ be a (d-1)-dimensional compact $C^{\infty}$-manifold without boundary, let $\mathcal{K}$ be a Hilbert space and let $K \in \mathcal{B}\left(\mathcal{K}, L^{2}(\Sigma)\right)$ with $\operatorname{ran} K \subset H^{r}(\Sigma)$, where $r>0$. Then $K$ is compact and $K \in \mathfrak{S}_{\frac{d-1}{r}, \infty}$.

2.2. Derivatives of holomorphic operator-valued functions. In the following we shall often use product rules for holomorphic operator-valued functions. Let $\mathcal{H}_{i}, i=1, \ldots, 4$, be Hilbert spaces, $\mathcal{U}$ a domain in $\mathbb{C}$ and let $A: \mathcal{U} \rightarrow \mathcal{B}\left(\mathcal{H}_{3}, \mathcal{H}_{4}\right)$, $B: \mathcal{U} \rightarrow \mathcal{B}\left(\mathcal{H}_{2}, \mathcal{H}_{3}\right), C: U \rightarrow \mathcal{B}\left(\mathcal{H}_{1}, \mathcal{H}_{2}\right)$ be holomorphic operator-valued functions. Then for $\lambda \in \mathcal{U}$ we have

$$
\begin{aligned}
\frac{\mathrm{d}^{m}}{\mathrm{~d} \lambda^{m}}(A(\lambda) B(\lambda)) & =\sum_{\substack{p+q=m \\
p, q \geq 0}}\left(\begin{array}{c}
m \\
p
\end{array}\right) A^{(p)}(\lambda) B^{(q)}(\lambda), \\
\frac{\mathrm{d}^{m}}{\mathrm{~d} \lambda^{m}}(A(\lambda) B(\lambda) C(\lambda)) & =\sum_{\substack{p+q+r=m \\
p, q, r \geq 0}} \frac{m !}{p ! q ! r !} A^{(p)}(\lambda) B^{(q)}(\lambda) C^{(r)}(\lambda) .
\end{aligned}
$$

If $A(\lambda)^{-1}$ is invertible for every $\lambda \in \mathcal{U}$, then relation (2.5a) implies the following formula for the derivative of the inverse,

$$
\frac{\mathrm{d}}{\mathrm{d} \lambda}\left(A(\lambda)^{-1}\right)=-A(\lambda)^{-1} A^{\prime}(\lambda) A(\lambda)^{-1} .
$$

2.3. Quasi boundary triples, Weyl functions and $\gamma$-fields. We begin this subsection by recalling the abstract concept of quasi boundary triples introduced in [8] as a generalization of the notion of (ordinary) boundary triples [16, 40]. For the theory of ordinary boundary triples and associated Weyl functions the reader may consult, e.g. [17, 21, 22]. Recent developments on quasi boundary triples and their applications to PDEs can be found in, e.g. [9, 11, 12, 13].

Definition 2.2. Let $S$ be a closed, densely defined, symmetric operator in a Hilbert space $\left(\mathcal{H},(\cdot, \cdot)_{\mathcal{H}}\right)$. A triple $\left\{\mathcal{G}, \Gamma_{0}, \Gamma_{1}\right\}$ is called a quasi boundary triple for $S^{*}$ if $\left(\mathcal{G},(\cdot, \cdot)_{\mathcal{G}}\right)$ is a Hilbert space, and for some linear operator $T \subset S^{*}$ with $\bar{T}=S^{*}$ the following assumptions are satisfied:

(i) $\Gamma_{0}, \Gamma_{1}: \operatorname{dom} T \rightarrow \mathcal{G}$ are linear mappings, and the mapping $\Gamma:=\left(\begin{array}{c}\Gamma_{0} \\ \Gamma_{1}\end{array}\right)$ has dense range in $\mathcal{G} \times \mathcal{G}$;

(ii) $A_{0}:=T\left\lceil\operatorname{ker} \Gamma_{0}\right.$ is a self-adjoint operator in $\mathcal{H}$; 
(iii) for all $f, g \in \operatorname{dom} T$ the abstract Green identity holds:

$$
(T f, g)_{\mathcal{H}}-(f, T g)_{\mathcal{H}}=\left(\Gamma_{1} f, \Gamma_{0} g\right)_{\mathcal{G}}-\left(\Gamma_{0} f, \Gamma_{1} g\right)_{\mathcal{G}} .
$$

Next, we recall the definitions of the $\gamma$-field and the Weyl function associated with a quasi boundary triple $\left\{\mathcal{G}, \Gamma_{0}, \Gamma_{1}\right\}$ for $S^{*}$. Note that the decomposition

$$
\operatorname{dom} T=\operatorname{dom} A_{0} \dot{+} \operatorname{ker}(T-\lambda)
$$

holds for all $\lambda \in \rho\left(A_{0}\right)$, so that $\Gamma_{0}\left\lceil\operatorname{ker}(T-\lambda)\right.$ is injective for all $\lambda \in \rho\left(A_{0}\right)$. The (operator-valued) functions $\gamma$ and $M$ defined by

$$
\gamma(\lambda):=\left(\Gamma_{0}\lceil\operatorname{ker}(T-\lambda))^{-1} \text { and } M(\lambda):=\Gamma_{1} \gamma(\lambda), \quad \lambda \in \rho\left(A_{0}\right),\right.
$$

are called the $\gamma$-field and the Weyl function corresponding to the quasi boundary triple $\left\{\mathcal{G}, \Gamma_{0}, \Gamma_{1}\right\}$. The adjoint of $\gamma(\bar{\lambda})$ has the following representation:

$$
\gamma(\bar{\lambda})^{*}=\Gamma_{1}\left(A_{0}-\lambda\right)^{-1}, \quad \lambda \in \rho\left(A_{0}\right) ;
$$

see $[8$, Proposition 2.6 (ii)] and also [9, Proposition 7.5]. According to [8, Proposition 2.6] the operator-valued functions $\lambda \mapsto \gamma(\lambda), \lambda \mapsto \gamma(\bar{\lambda})^{*}$ and $\lambda \mapsto M(\lambda)$ are holomorphic on $\rho\left(A_{0}\right)$. Finally, we recall formulae for their derivatives: for $k \in \mathbb{N}$, $\varphi \in \operatorname{ran} \Gamma_{0}$ and $\lambda \in \rho\left(A_{0}\right)$ we have

$$
\begin{aligned}
\gamma^{(k)}(\lambda) \varphi & =k !\left(A_{0}-\lambda\right)^{-k} \gamma(\lambda) \varphi \\
\frac{\mathrm{d}^{k}}{\mathrm{~d} \lambda^{k}}(\gamma(\bar{\lambda}))^{*} & =k ! \gamma(\bar{\lambda})^{*}\left(A_{0}-\lambda\right)^{-k}, \\
M^{(k)}(\lambda) \varphi & =k ! \gamma(\bar{\lambda})^{*}\left(A_{0}-\lambda\right)^{-(k-1)} \gamma(\lambda) \varphi ;
\end{aligned}
$$

see [12, Lemma 2.4].

2.4. Self-adjoint extensions and abstract Krein's resolvent formula. In this subsection we parameterize subfamilies of self-adjoint extensions via quasi boundary triples and provide a couple of useful Krein-type formulae for resolvent differences of these extensions.

The following hypothesis will be useful in the following.

Hypothesis 2.1. Let $S$ be a closed, densely defined, symmetric operator in a Hilbert space $\mathcal{H}$ and let $\left\{\mathcal{G}, \Gamma_{0}, \Gamma_{1}\right\}$ be a quasi boundary triple for $S^{*}$ such that $\operatorname{ran} \Gamma_{0}=$ G. Moreover, let $\gamma$ and $M$ be the associated $\gamma$-field and Weyl function, respectively.

We remark that the quasi boundary triple $\left\{\mathcal{G}, \Gamma_{0}, \Gamma_{1}\right\}$ in Hypothesis 2.1 is also a generalized boundary triple in the sense of [22]. In this case the $\gamma$-field and the Weyl function associated with $\left\{\mathcal{G}, \Gamma_{0}, \Gamma_{1}\right\}$ are defined on the whole space $\mathcal{G}$, and the formulae (2.8a) and (2.8c) are valid for all $\varphi \in \mathcal{G}$.

Next, we state a Krein-type formula for the resolvent difference of $A_{j}:=T \uparrow$ $\operatorname{ker} \Gamma_{j}, j=0,1$. 
Proposition 2.3. [12, Theorem 2.5] Assume that Hypothesis 2.1 is satisfied and that $A_{1}$ is self-adjoint in $\mathcal{H}$. Then the formula

$$
\left(A_{0}-\lambda\right)^{-1}-\left(A_{1}-\lambda\right)^{-1}=\gamma(\lambda) M(\lambda)^{-1} \gamma(\bar{\lambda})^{*}
$$

holds for all $\lambda \in \rho\left(A_{0}\right) \cap \rho\left(A_{1}\right)$.

In the next proposition, we formulate a sufficient condition for self-adjointness of the extension of $A$ defined by

$$
A_{[B]}:=T \uparrow \operatorname{ker}\left(B \Gamma_{1}-\Gamma_{0}\right),
$$

and provide a Krein-type formula for the resolvent difference of $A_{[B]}$ and $A_{0}$.

Proposition 2.4. [12, Theorem 2.6] Assume that Hypothesis 2.1 is satisfied, that $M\left(\lambda_{0}\right) \in \mathfrak{S}_{\infty}(\mathcal{G})$ for some $\lambda_{0} \in \rho\left(A_{0}\right)$, and that $B \in \mathcal{B}(\mathcal{G})$ is self-adjoint in $\mathcal{G}$. Then the extension $A_{[B]}$ of $A$ is self-adjoint in $\mathcal{H}$, and the formula

$$
\left(A_{[B]}-\lambda\right)^{-1}-\left(A_{0}-\lambda\right)^{-1}=\gamma(\lambda)(I-B M(\lambda))^{-1} B \gamma(\bar{\lambda})^{*}
$$

holds for all $\lambda \in \rho\left(A_{[B]}\right) \cap \rho\left(A_{0}\right)$. In this formula the middle term satisfies

$$
(I-B M(\lambda))^{-1} \in \mathcal{B}(\mathcal{G})
$$

for all $\lambda \in \rho\left(A_{[B]}\right) \cap \rho\left(A_{0}\right)$.

2.5. Quasi boundary triples for coupled problems. We recall particular quasi boundary triples, which are used to parameterize the self-adjoint operators from Definition 1.1. Furthermore, we reformulate some of the abstract statements from Subsections 2.3 and 2.4 for these quasi boundary triples.

First, we introduce the subspace $H_{\Delta}^{3 / 2}\left(\mathbb{R}^{d} \backslash \Sigma\right)$ of $L^{2}\left(\mathbb{R}^{d}\right)$ by

$$
H_{\Delta}^{3 / 2}\left(\mathbb{R}^{d} \backslash \Sigma\right):=H_{\Delta}^{3 / 2}\left(\Omega_{+}\right) \oplus H_{\Delta}^{3 / 2}\left(\Omega_{-}\right)
$$

where $H_{\Delta}^{3 / 2}\left(\Omega_{ \pm}\right)$are as in (1.3). Further, to shorten the notations, we also define the jump of the normal derivative by $\left[\partial_{\nu} u\right]_{\Sigma}:=\left.\partial_{\nu_{+}} u_{+}\right|_{\Sigma}+\left.\partial_{\nu_{-}} u_{-}\right|_{\Sigma}$ for $u \in H_{\Delta}^{3 / 2}\left(\mathbb{R}^{d} \backslash \Sigma\right)$. Following the lines of [10, Section 3], we define the operators $\widetilde{T}$ and $\widehat{T}$ in $L^{2}\left(\mathbb{R}^{d}\right)$ by

$$
\begin{array}{ll}
\widetilde{T} u:=\left(-\Delta u_{+}\right) \oplus\left(-\Delta u_{-}\right), & \operatorname{dom} \widetilde{T}:=\left\{u \in H_{\Delta}^{3 / 2}\left(\mathbb{R}^{d} \backslash \Sigma\right):[u]_{\Sigma}=0\right\}, \\
\widehat{T} u:=\left(-\Delta u_{+}\right) \oplus\left(-\Delta u_{-}\right), & \operatorname{dom} \widehat{T}:=\left\{u \in H_{\Delta}^{3 / 2}\left(\mathbb{R}^{d} \backslash \Sigma\right):\left[\partial_{\nu} u\right]_{\Sigma}=0\right\},
\end{array}
$$

and their restrictions $\widetilde{S}$ and $\widehat{S}$ by

$$
\begin{aligned}
& \widetilde{S}:=\widetilde{T} \uparrow\left\{u \in H_{\Delta}^{3 / 2}\left(\mathbb{R}^{d} \backslash \Sigma\right):\left.u_{ \pm}\right|_{\Sigma}=0,\left[\partial_{\nu} u\right]_{\Sigma}=0\right\}, \\
& \widehat{S}:=\widehat{T} \uparrow\left\{u \in H_{\Delta}^{3 / 2}\left(\mathbb{R}^{d} \backslash \Sigma\right):\left.\partial_{\nu_{ \pm}} u_{ \pm}\right|_{\Sigma}=0,[u]_{\Sigma}=0\right\} .
\end{aligned}
$$


It can be verified that $\widetilde{S}$ (respectively, $\widehat{S}$ ) is the restriction of $\mathrm{H}_{\text {free }}$ to functions, whose Dirichlet trace (respectively, Neumann trace) vanishes on $\Sigma$. In particular, as a consequence of this identification we arrive at the inclusions $\operatorname{dom} \widetilde{S}, \operatorname{dom} \widehat{S} \subset$ $H^{2}\left(\mathbb{R}^{d}\right)$. It can also be shown that the operators $\widetilde{S}$ and $\widehat{S}$ are closed, densely defined, and symmetric in $L^{2}\left(\mathbb{R}^{d}\right)$ and that the closures of $\widetilde{T}$ and $\widehat{T}$ coincide with $\widetilde{S}^{*}$ and $\widehat{S}^{*}$, respectively. Furthermore, we define the boundary mappings by

$$
\begin{array}{lll}
\widetilde{\Gamma}_{0}, \widetilde{\Gamma}_{1}: \operatorname{dom} \widetilde{T} \rightarrow L^{2}(\Sigma), & \widetilde{\Gamma}_{0} u:=\left[\partial_{\nu} u\right]_{\Sigma}, & \widetilde{\Gamma}_{1} u:=\left.u\right|_{\Sigma}, \\
\widehat{\Gamma}_{0}, \widehat{\Gamma}_{1}: \operatorname{dom} \widehat{T} \rightarrow L^{2}(\Sigma), & \widehat{\Gamma}_{0} u:=\left.\partial_{\nu_{+}} u_{+}\right|_{\Sigma}, & \widehat{\Gamma}_{1} u:=[u]_{\Sigma}
\end{array}
$$

The identities

$$
\mathrm{H}_{\text {free }}=\widetilde{T} \uparrow \operatorname{ker} \widetilde{\Gamma}_{0}=\widehat{T} \uparrow \operatorname{ker} \widehat{\Gamma}_{1} \quad \text { and } \quad \mathrm{K}_{\mathrm{N}}=\widehat{T} \uparrow \operatorname{ker} \widehat{\Gamma}_{0}
$$

can be checked in a straightforward way. According to [10, Proposition 3.2 (i)] the triple $\widetilde{\Pi}:=\left\{L^{2}(\Sigma), \widetilde{\Gamma}_{0}, \widetilde{\Gamma}_{1}\right\}$ is a quasi boundary triple for $\widetilde{S}^{*}$, and by $[10$, Proposition $3.8(\mathrm{i})]$ the triple $\widehat{\Pi}:=\left\{L^{2}(\Sigma), \widehat{\Gamma}_{0}, \widehat{\Gamma}_{1}\right\}$ is a quasi boundary triple for $\widehat{S}^{*}$.

Definition 2.5. Let $\widetilde{\gamma}, \widetilde{M}$ and $\widehat{\gamma}, \widehat{M}$ be the $\gamma$-fields and the Weyl functions of the quasi boundary triples $\widetilde{\Pi}$ and $\widehat{\Pi}$, respectively.

Remark 2.6. The definitions of the operator-valued functions $\widetilde{M}$ and $\widehat{M}$ as Neumannto-Dirichlet maps in (1.4) and as Weyl functions of the quasi boundary triples $\widetilde{\Pi}$ and $\widehat{\Pi}$ are equivalent; see [10, Propositions 3.2 (iii) and 3.8 (iii)].

Remark 2.7. According to [10, Propositions 3.2 (ii) and 3.8 (ii)], for any $\varphi \in L^{2}(\Sigma)$ both transmission boundary value problems

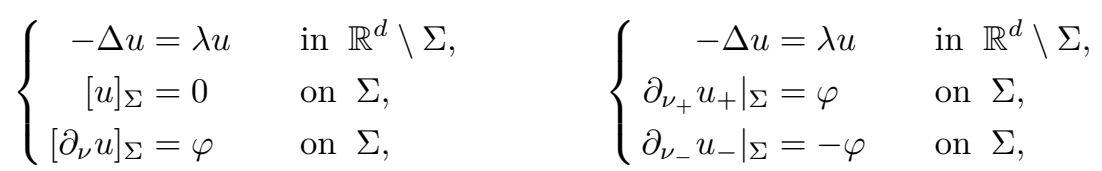

have unique solutions $\widetilde{u}(\varphi), \widehat{u}(\varphi) \in H_{\Delta}^{3 / 2}\left(\mathbb{R}^{d} \backslash \Sigma\right)$. Moreover, the operator-valued functions $\widetilde{\gamma}$ and $\widehat{\gamma}$ satisfy $\widetilde{\gamma}(\lambda) \varphi=\widetilde{u}_{\lambda}(\varphi)$ and $\widehat{\gamma}(\lambda) \varphi=\widehat{u}_{\lambda}(\varphi)$ for $\varphi \in L^{2}(\Sigma)$ and $\lambda \in \mathbb{C} \backslash \mathbb{R}_{+}$.

Thanks to (2.7) the adjoints of $\widetilde{\gamma}(\bar{\lambda})$ and $\widehat{\gamma}(\bar{\lambda})$ can be expressed as

$$
\widetilde{\gamma}(\bar{\lambda})^{*}=\widetilde{\Gamma}_{1}\left(\mathrm{H}_{\text {free }}-\lambda\right)^{-1} \quad \text { and } \quad \widehat{\gamma}(\bar{\lambda})^{*}=\widehat{\Gamma}_{1}\left(\mathrm{~K}_{\mathrm{N}}-\lambda\right)^{-1}
$$

for $\lambda \in \mathbb{C} \backslash \mathbb{R}_{+}$. We also remark that, by [10, Propositions 3.2 (iii) and 3.8 (iii)], we have

$$
\operatorname{ran} \widetilde{M}(\lambda)=\operatorname{ran} \widehat{M}(\lambda)=H^{1}(\Sigma), \quad \lambda \in \mathbb{C} \backslash \mathbb{R}_{+} .
$$

According to Proposition 2.3, the formula

$$
\left(\mathrm{K}_{\mathrm{N}}-\lambda\right)^{-1}-\left(\mathrm{H}_{\text {free }}-\lambda\right)^{-1}=\widehat{\gamma}(\lambda) \widehat{M}(\lambda)^{-1} \widehat{\gamma}(\bar{\lambda})^{*},
$$


holds for all $\lambda \in \mathbb{C} \backslash \mathbb{R}_{+}$. Since the operators of multiplication with $\alpha$ and $\omega$ are bounded and self-adjoint in $L^{2}(\Sigma)$, by Proposition 2.4 the extensions

$$
\widetilde{T} \uparrow \operatorname{ker}\left(\alpha \widetilde{\Gamma}_{1}-\widetilde{\Gamma}_{0}\right) \quad \text { and } \quad \widehat{T} \uparrow \operatorname{ker}\left(\omega \widehat{\Gamma}_{1}-\widehat{\Gamma}_{0}\right)
$$

are self-adjoint in $L^{2}\left(\mathbb{R}^{d}\right)$. In a way similar to [10], one can check that these restrictions coincide with $\mathrm{H}_{\alpha, \Sigma}$ and $\mathrm{K}_{\omega, \Sigma}$, respectively. Moreover, by Proposition 2.4, the formulae

$$
\begin{aligned}
\left(\mathrm{H}_{\alpha, \Sigma}-\lambda\right)^{-1}-\left(\mathrm{H}_{\text {free }}-\lambda\right)^{-1} & =\widetilde{\gamma}(\lambda)(I-\alpha \widetilde{M}(\lambda))^{-1} \alpha \widetilde{\gamma}(\bar{\lambda})^{*}, \\
\left(\mathrm{~K}_{\omega, \Sigma}-\lambda\right)^{-1}-\left(\mathrm{K}_{\mathrm{N}}-\lambda\right)^{-1} & =\widehat{\gamma}(\lambda)(I-\omega \widehat{M}(\lambda))^{-1} \omega \widehat{\gamma}(\bar{\lambda})^{*},
\end{aligned}
$$

hold for all $\lambda \in \rho\left(\mathrm{H}_{\alpha, \Sigma}\right)$ and all $\lambda \in \rho\left(\mathrm{K}_{\omega, \Sigma}\right)$, respectively. In these formulae the middle terms on the right-hand sides satisfy

$$
(I-\alpha \widetilde{M}(\lambda))^{-1},(I-\omega \widehat{M}(\lambda))^{-1} \in \mathcal{B}\left(L^{2}(\Sigma)\right)
$$

for $\lambda$ in the respective resolvent sets.

\section{Proofs of the main results}

In this section we prove the main results of the paper: the trace formulae for the Schrödinger operators with singular interactions. Theorems 1.2 and 1.3 are proved in Subsections 3.1 and 3.2, respectively. Throughout this section we use the notations $R(\lambda):=\left(\mathrm{H}_{\text {free }}-\lambda\right)^{-1}$ and $R_{\mathrm{N}}(\lambda):=\left(\mathrm{K}_{\mathrm{N}}-\lambda\right)^{-1}$.

3.1. Proof of Theorem 1.2. To prove Theorem 1.2 we need an auxiliary lemma.

Lemma 3.1. Let the $\gamma$-field $\widetilde{\gamma}$ and the Weyl function $\widetilde{M}$ be as in Definition 2.5. Then for every $\lambda \in \mathbb{C} \backslash \mathbb{R}_{+}$and every $k \in \mathbb{N}_{0}$ the following relations hold:

(i) $\widetilde{\gamma}^{(k)}(\lambda), \frac{\mathrm{d}^{k}}{\mathrm{~d} \lambda^{k}} \widetilde{\gamma}(\bar{\lambda})^{*} \in \mathfrak{S}_{\frac{d-1}{2 k+3 / 2}, \infty} ;$

(ii) $\widetilde{M}^{(k)}(\lambda) \in \mathfrak{S}_{\frac{d-1}{2 k+1}, \infty}$.

Proof. (i) Let $\lambda \in \mathbb{C} \backslash \mathbb{R}_{+}$and $k \in \mathbb{N}_{0}$. First, we observe that $\operatorname{ran}\left(R(\lambda)^{k}\right) \subset$ $H^{2 k}\left(\mathbb{R}^{d}\right)$. By the trace theorem we have $\left.u\right|_{\Sigma} \in H^{s-1 / 2}(\Sigma)$ for every $u \in H^{s}\left(\mathbb{R}^{d}\right)$ with $s>1 / 2$. Hence, we obtain from (2.11) that

$$
\operatorname{ran}\left(\widetilde{\gamma}(\bar{\lambda})^{*} R(\lambda)^{k}\right) \subset H^{2 k+3 / 2}(\Sigma) .
$$

Thus Lemma 2.1 with $\mathcal{K}=L^{2}\left(\mathbb{R}^{d}\right)$ and $r=2 k+3 / 2$ implies that

$$
\widetilde{\gamma}(\bar{\lambda})^{*} R(\lambda)^{k} \in \mathfrak{S}_{\frac{d-1}{2 k+3 / 2}, \infty} .
$$

By taking the adjoint in (3.1) and replacing $\lambda$ by $\bar{\lambda}$ we obtain

$$
R(\lambda)^{k} \widetilde{\gamma}(\lambda) \in \mathfrak{S}_{\frac{d-1}{2 k+3 / 2}, \infty} .
$$


From (2.8a), (2.8b), (3.1) and (3.2) we now obtain $\widetilde{\gamma}^{(k)}(\lambda), \frac{\mathrm{d}^{k}}{\mathrm{~d} \lambda^{k}} \widetilde{\gamma}(\bar{\lambda})^{*} \in \mathfrak{S}_{\frac{d-1}{2 k+3 / 2}, \infty}$.

(ii) For $k=0$ we observe that by $(2.12)$ we have $\operatorname{ran} \widetilde{M}(\lambda)=H^{1}(\Sigma)$. Therefore, Lemma 2.1 with $\mathcal{K}=L^{2}(\Sigma)$ and $r=1$ implies that $\widetilde{M}(\lambda) \in \mathfrak{S}_{d-1, \infty}$. For $k \geq 1$ we derive from $(2.8 \mathrm{c})$ that

$$
\widetilde{M}^{(k)}(\lambda)=k ! \widetilde{\gamma}(\bar{\lambda})^{*} R(\lambda)^{k-1} \widetilde{\gamma}(\lambda) \in \mathfrak{S}_{\frac{d-1}{2(k-1)+3 / 2}, \infty} \cdot \mathfrak{S}_{\frac{d-1}{3 / 2}, \infty}=\mathfrak{S}_{\frac{d-1}{2 k+1}, \infty},
$$

where we applied (3.1), (3.2) and (2.2).

Proof of Theorem 1.2. In order to shorten notation and to avoid the distinction of several cases, we set

$$
\mathfrak{A}_{r}:= \begin{cases}\mathfrak{S}_{\frac{d-1}{r}, \infty}\left(L^{2}(\Sigma)\right) & \text { if } r>0, \\ \mathcal{B}\left(L^{2}(\Sigma)\right) & \text { if } r=0 .\end{cases}
$$

It follows from $(2.2)$ and the fact that $\mathfrak{S}_{p, \infty}\left(L^{2}(\Sigma)\right)$ is an ideal in $\mathcal{B}\left(L^{2}(\Sigma)\right)$ for $p>0$ that

$$
\mathfrak{A}_{r_{1}} \cdot \mathfrak{A}_{r_{2}}=\mathfrak{A}_{r_{1}+r_{2}}, \quad r_{1}, r_{2} \geq 0 .
$$

The remainder of the proof is divided into two steps.

Step 1. Let $\alpha \in L^{\infty}(\Sigma ; \mathbb{R})$ and set

$$
\widetilde{T}(\lambda):=(I-\alpha \widetilde{M}(\lambda))^{-1}, \quad \lambda \in \rho\left(\mathrm{H}_{\alpha, \Sigma}\right),
$$

where $\widetilde{T}(\lambda) \in \mathcal{B}\left(L^{2}(\Sigma)\right)$ by $(2.15)$. Next, we show that

$$
\widetilde{T}^{(k)}(\lambda) \in \mathfrak{A}_{2 k+1}, \quad k \in \mathbb{N},
$$

by induction. Relation (2.6) implies that

$$
\widetilde{T}^{\prime}(\lambda)=\widetilde{T}(\lambda) \alpha \widetilde{M}^{\prime}(\lambda) \widetilde{T}(\lambda),
$$

which is in $\mathfrak{A}_{3}$ by Lemma 3.1 (ii). Let $m \in \mathbb{N}$ and assume that (3.4) is true for every $k=1, \ldots, m$, which implies, in particular, that

$$
\widetilde{T}^{(k)}(\lambda) \in \mathfrak{A}_{2 k}, \quad k=0, \ldots, m .
$$

Then

$$
\begin{aligned}
\widetilde{T}^{(m+1)}(\lambda) & =\frac{\mathrm{d}^{m}}{\mathrm{~d} \lambda^{m}}\left(\widetilde{T}(\lambda) \alpha \widetilde{M}^{\prime}(\lambda) \widetilde{T}(\lambda)\right) \\
& =\sum_{\substack{p+q+r=m \\
p, q, r \geq 0}} \frac{m !}{p ! q ! r !} \widetilde{T}^{(p)}(\lambda) \alpha \widetilde{M}^{(q+1)}(\lambda) \widetilde{T}^{(r)}(\lambda)
\end{aligned}
$$


by (3.5) and (2.5b). Relation (3.6), the boundedness of $\alpha$, Lemma 3.1 (ii) and (3.3) imply that

$$
\widetilde{T}^{(p)}(\lambda) \alpha \widetilde{M}^{(q+1)}(\lambda) \widetilde{T}^{(r)}(\lambda) \in \mathfrak{A}_{2 p} \cdot \mathfrak{A}_{2(q+1)+1} \cdot \mathfrak{A}_{2 r}=\mathfrak{A}_{2(m+1)+1},
$$

since $p+q+r=m$. This shows (3.4) for $k=m+1$ and hence, by induction, for all $k \in \mathbb{N}$. Since $\widetilde{T}(\lambda) \in \mathcal{B}\left(L^{2}(\Sigma)\right)$, we have, in particular,

$$
\widetilde{T}^{(k)}(\lambda) \in \mathfrak{A}_{2 k}, \quad k \in \mathbb{N}_{0}, \lambda \in \rho\left(\mathrm{H}_{\alpha, \Sigma}\right) .
$$

Step 2. By taking derivatives we obtain from (2.14a) that, for $m \in \mathbb{N}$,

$$
\begin{aligned}
(m-1) ! \widetilde{D}_{\alpha, m}(\lambda) & =\frac{\mathrm{d}^{m-1}}{\mathrm{~d} \lambda^{m-1}}\left(\widetilde{D}_{\alpha, 1}(\lambda)\right)=\frac{\mathrm{d}^{m-1}}{\mathrm{~d} \lambda^{m-1}}\left(\widetilde{\gamma}(\lambda) \widetilde{T}(\lambda) \alpha \widetilde{\gamma}(\bar{\lambda})^{*}\right) \\
& =\sum_{\substack{p+q+r=m-1 \\
p, q, r \geq 0}} \frac{(m-1) !}{p ! q ! r !} \widetilde{\gamma}^{(p)}(\lambda) \widetilde{T}^{(q)}(\lambda) \alpha \frac{\mathrm{d}^{r}}{\mathrm{~d} \lambda^{r}} \widetilde{\gamma}(\bar{\lambda})^{*}
\end{aligned}
$$

By Lemma 3.1(i) and (3.7), each term in the sum satisfies

$$
\widetilde{\gamma}^{(p)}(\lambda) \widetilde{T}^{(q)}(\lambda) \alpha \frac{\mathrm{d}^{r}}{\mathrm{~d} \lambda^{r}} \widetilde{\gamma}(\bar{\lambda})^{*} \in \mathfrak{A}_{2 p+3 / 2} \cdot \mathfrak{A}_{2 q} \cdot \mathfrak{A}_{2 r+3 / 2}=\mathfrak{A}_{2 m+1}=\mathfrak{S}_{\frac{d-1}{2 m+1}, \infty} .
$$

If $m \in \mathbb{N}$ is such that $m>\frac{d-2}{2}$, then $\frac{d-1}{2 m+1}<1$ and, by (2.1) and (3.9), all terms in the sum in (3.8) are trace class operators, and the same is true if we change the order in the product in (3.9). Hence, we can apply the trace to the expression in (3.8) and use (2.3), (2.4) and (2.8c) to obtain

$$
\begin{aligned}
& (m-1) ! \operatorname{Tr}\left(\widetilde{D}_{\alpha, m}(\lambda)\right)=\operatorname{Tr}\left(\sum_{\substack{p+q+r=m-1 \\
p, q, r \geq 0}} \frac{(m-1) !}{p ! q ! r !} \widetilde{\gamma}^{(p)}(\lambda) \widetilde{T}^{(q)}(\lambda) \alpha \frac{\mathrm{d}^{r}}{\mathrm{~d} \lambda^{r}} \widetilde{\gamma}(\bar{\lambda})^{*}\right) \\
& =\sum_{\substack{p+q+r=m-1 \\
p, q, r \geq 0}} \frac{(m-1) !}{p ! q ! r !} \operatorname{Tr}\left(\widetilde{\gamma}^{(p)}(\lambda) \widetilde{T}^{(q)}(\lambda) \alpha \frac{\mathrm{d}^{r}}{\mathrm{~d} \lambda^{r}} \widetilde{\gamma}(\bar{\lambda})^{*}\right) \\
& =\sum_{\substack{p+q+r=m-1 \\
p, q, r \geq 0}} \frac{(m-1) !}{p ! q ! r !} \operatorname{Tr}\left(\widetilde{T}^{(q)}(\lambda) \alpha\left(\frac{\mathrm{d}^{r}}{\mathrm{~d} \lambda^{r}} \widetilde{\gamma}(\bar{\lambda})^{*}\right) \widetilde{\gamma}^{(p)}(\lambda)\right) \\
& =\operatorname{Tr}\left(\sum_{\substack{p+q+r=m-1 \\
p, q, r \geq 0}} \frac{(m-1) !}{p ! q ! r !} \widetilde{T}^{(q)}(\lambda) \alpha\left(\frac{\mathrm{d}^{r}}{\mathrm{~d} \lambda^{r}} \widetilde{\gamma}(\bar{\lambda})^{*}\right) \widetilde{\gamma}^{(p)}(\lambda)\right) \\
& =\operatorname{Tr}\left(\frac{\mathrm{d}^{m-1}}{\mathrm{~d} \lambda^{m-1}}\left(\widetilde{T}(\lambda) \alpha \widetilde{\gamma}(\bar{\lambda})^{*} \widetilde{\gamma}(\lambda)\right)\right)=\operatorname{Tr}\left(\frac{\mathrm{d}^{m-1}}{\mathrm{~d} \lambda^{m-1}}\left(\widetilde{T}(\lambda) \alpha \widetilde{M}^{\prime}(\lambda)\right)\right)
\end{aligned}
$$

which finishes the proof. 
3.2. Proof of Theorem 1.3. First, we need three preparatory lemmas. The proof of the first of them is completely analogous to the proof of Lemma 3.1 and is therefore omitted.

Lemma 3.2. Let the $\gamma$-field $\widehat{\gamma}$ and the Weyl function $\widehat{M}$ be as in Definition 2.5. Then for every $\lambda \in \mathbb{C} \backslash \mathbb{R}_{+}$and every $k \in \mathbb{N}_{0}$ the following relations hold:

(i) $\widehat{\gamma}^{(k)}(\lambda), \frac{\mathrm{d}^{k}}{\mathrm{~d} \lambda^{k}} \widehat{\gamma}(\bar{\lambda})^{*} \in \mathfrak{S}_{\frac{d-1}{2 k+3 / 2}, \infty}$;

(ii) $\widehat{M}^{(k)}(\lambda) \in \mathfrak{S}_{\frac{d-1}{2 k+1}, \infty}$.

Lemma 3.3. Let the $\gamma$-field $\widehat{\gamma}$ and the Weyl function $\widehat{M}$ be as in Definition 2.5. Then for all $s \geq 0$, and all $\lambda \in \mathbb{C} \backslash \mathbb{R}_{+}$the following statements hold:

(i) $\operatorname{ran}\left(\widehat{\gamma}(\bar{\lambda})^{*} \uparrow H^{s}\left(\mathbb{R}^{d}\right)\right) \subset H^{s+\frac{3}{2}}(\Sigma)$;

(ii) $\operatorname{ran}\left(\widehat{M}(\lambda) \uparrow H^{s}(\Sigma)\right)=H^{s+1}(\Sigma)$.

Proof. (i) According to (2.11) we have

$$
\widehat{\gamma}(\bar{\lambda})^{*}=\widehat{\Gamma}_{1} R_{\mathrm{N}}(\lambda)
$$

Employing the regularity shift property [45, Theorem 4.20] and the trace theorem [45, Theorem 3.37] we conclude that

$$
\operatorname{ran}\left(\widehat{\gamma}(\bar{\lambda})^{*}\left\lceil H^{s}\left(\mathbb{R}^{d}\right)\right) \subset H^{s+\frac{3}{2}}(\Sigma)\right.
$$

holds for all $s \geq 0$.

(ii) Define the space $H^{s}\left(\mathbb{R}^{d} \backslash \Sigma\right):=H^{s}\left(\Omega_{+}\right) \oplus H^{s}\left(\Omega_{-}\right)$. It follows from the decomposition $\operatorname{dom} \widehat{T}=\operatorname{dom} \mathrm{K}_{\mathrm{N}} \dot{+} \operatorname{ker}(\widehat{T}-\lambda), \lambda \in \mathbb{C} \backslash \mathbb{R}_{+}$, and the properties of the Neumann trace $[43, \S 2.7 .3]$ that the restriction of the mapping $\widehat{\Gamma}_{0}$ to

$$
\operatorname{ker}(\widehat{T}-\lambda) \cap H^{s+\frac{3}{2}}\left(\mathbb{R}^{d} \backslash \Sigma\right)
$$

is a bijection onto $H^{s}(\Sigma)$ for $s \geq 0$. This, together with the definition of the $\gamma$-field, implies that

$$
\operatorname{ran}\left(\widehat{\gamma}(\lambda) \uparrow H^{s}(\Sigma)\right)=\operatorname{ker}(\widehat{T}-\lambda) \cap H^{s+\frac{3}{2}}\left(\mathbb{R}^{d} \backslash \Sigma\right) \subset H^{s+\frac{3}{2}}\left(\mathbb{R}^{d} \backslash \Sigma\right) .
$$

Hence, it follows from the definition of $\widehat{M}(\lambda)$, the definition of $\widehat{\Gamma}_{1}$ in $(2.10)$ and the trace theorem that

$$
\operatorname{ran}\left(\widehat{M}(\lambda) \uparrow H^{s}(\Sigma)\right) \subset H^{s+1}(\Sigma) .
$$

To verify the opposite inclusion, let $\psi \in H^{s+1}(\Sigma)$. The decomposition $\operatorname{dom} \widehat{T}=$ $\operatorname{dom} \mathrm{H}_{\text {free }} \dot{+} \operatorname{ker}(\widehat{T}-\lambda), \lambda \in \mathbb{C} \backslash \mathbb{R}_{+}$implies that there exists a function $f_{\lambda} \in$ $\operatorname{ker}(\widehat{T}-\lambda) \cap H^{s+\frac{3}{2}}\left(\mathbb{R}^{d} \backslash \Sigma\right)$ such that $\widehat{\Gamma}_{1} f_{\lambda}=\psi$. Thus,

$$
\widehat{\Gamma}_{0} f_{\lambda}=\varphi \in H^{s}(\Sigma) \quad \text { and } \quad \widehat{M}(\lambda) \varphi=\psi,
$$

that is, $H^{s+1}(\Sigma) \subset \operatorname{ran}\left(\widehat{M}(\lambda) \uparrow H^{s}(\Sigma)\right)$, and the assertion is shown. 
Lemma 3.4. Let the self-adjoint operators $\mathrm{H}_{\text {free }}$ and $\mathrm{K}_{\mathrm{N}}$ be as in Definition 1.1, and let the operator-valued function $\widehat{M}$ be as in (1.4). Then for all $m \in \mathbb{N}$ such that $m>\frac{d-1}{2}$ and all $\lambda \in \mathbb{C} \backslash \mathbb{R}_{+}$the resolvent power difference

$$
\widehat{D}_{m}(\lambda):=\left(\mathrm{K}_{\mathrm{N}}-\lambda\right)^{-m}-\left(\mathrm{H}_{\text {free }}-\lambda\right)^{-m}
$$

belongs to the trace class, and its trace can be expressed as

$$
\operatorname{Tr}\left(\widehat{D}_{m}(\lambda)\right)=\frac{1}{(m-1) !} \operatorname{Tr}\left(\frac{\mathrm{d}^{m-1}}{\mathrm{~d} \lambda^{m-1}}\left(\widehat{M}(\lambda)^{-1} \widehat{M}^{\prime}(\lambda)\right)\right)
$$

Proof. The proof is divided into three steps.

Step 1. Let us introduce the operator-valued function

$$
S(\lambda):=\widehat{M}(\lambda)^{-1} \widehat{\gamma}(\bar{\lambda})^{*}, \quad \lambda \in \mathbb{C} \backslash \mathbb{R}_{+} .
$$

Note that the product is well defined since by Lemma 3.3 (i)

$$
\operatorname{ran}\left(\widehat{\gamma}(\bar{\lambda})^{*}\right) \subset H^{1}(\Sigma)=\operatorname{dom}\left(\widehat{M}(\lambda)^{-1}\right) .
$$

The closed graph theorem implies that $S(\lambda) \in \mathcal{B}\left(L^{2}\left(\mathbb{R}^{d}\right), L^{2}(\Sigma)\right)$ for all $\lambda \in \mathbb{C} \backslash \mathbb{R}_{+}$. Next we prove the following smoothing property for the derivatives of $S$ :

$$
\operatorname{ran}\left(S^{(k)}(\lambda) \uparrow H^{s}\left(\mathbb{R}^{d}\right)\right) \subset H^{s+2 k+1 / 2}(\Sigma), \quad s \geq 0, k \in \mathbb{N}_{0},
$$

by induction. Since, by Lemma $3.3(\mathrm{i}), \widehat{\gamma}(\bar{\lambda})^{*}$ maps $H^{s}\left(\mathbb{R}^{d}\right)$ into $H^{s+3 / 2}(\Sigma)$ for all $s \geq 0$ and $\widehat{M}(\lambda)^{-1}$ maps $H^{s+3 / 2}(\Sigma)$ into $H^{s+1 / 2}(\Sigma)$ by Lemma 3.3 (ii), relation (3.10) is true for $k=0$. Now let $l \in \mathbb{N}_{0}$ and assume that (3.10) is true for every $k=0,1, \ldots, l$. It follows from $(2.5 \mathrm{a}),(2.6),(2.8 \mathrm{~b}),(2.8 \mathrm{c})$ and $(2.13)$ that for $\lambda \in \mathbb{C} \backslash \mathbb{R}_{+}$,

$$
\begin{aligned}
S^{\prime}(\lambda) & =\frac{\mathrm{d}}{\mathrm{d} \lambda}\left(\widehat{M}(\lambda)^{-1}\right) \widehat{\gamma}(\bar{\lambda})^{*}+\widehat{M}(\lambda)^{-1} \frac{\mathrm{d}}{\mathrm{d} \lambda} \widehat{\gamma}(\bar{\lambda})^{*} \\
& =-\widehat{M}(\lambda)^{-1} \widehat{M}^{\prime}(\lambda) \widehat{M}(\lambda)^{-1} \widehat{\gamma}(\bar{\lambda})^{*}+\widehat{M}(\lambda)^{-1} \widehat{\gamma}(\bar{\lambda})^{*} R_{\mathrm{N}}(\lambda) \\
& =-\widehat{M}(\lambda)^{-1} \widehat{\gamma}(\bar{\lambda})^{*} \widehat{\gamma}(\lambda) \widehat{M}(\lambda)^{-1} \widehat{\gamma}(\bar{\lambda})^{*}+\widehat{M}(\lambda)^{-1} \widehat{\gamma}(\bar{\lambda})^{*} R_{\mathrm{N}}(\lambda) \\
& =S(\lambda)\left[R_{\mathrm{N}}(\lambda)-\widehat{\gamma}(\lambda) \widehat{M}(\lambda)^{-1} \widehat{\gamma}(\bar{\lambda})^{*}\right]=S(\lambda) R(\lambda)
\end{aligned}
$$

Hence, with the help of $(2.5 \mathrm{a})$ we obtain

$$
\begin{aligned}
S^{(l+1)}(\lambda) & =\frac{\mathrm{d}^{l}}{\mathrm{~d} \lambda^{l}}(S(\lambda) R(\lambda))=\sum_{\substack{p+q=l \\
p, q \geq 0}}\left(\begin{array}{l}
l \\
p
\end{array}\right) S^{(p)}(\lambda) R^{(q)}(\lambda) \\
& =\sum_{\substack{p+q=l \\
p, q \geq 0}} \frac{l !}{p !} S^{(p)}(\lambda) R(\lambda)^{q+1}
\end{aligned}
$$


Using the induction hypothesis, formula (3.11) and smoothing properties of $R(\lambda)$, we deduce that, for $p, q \geq 0, p+q=l$,

$$
\begin{aligned}
\operatorname{ran}\left(S^{(p)}(\lambda) R(\lambda)^{q+1}\left\lceil H^{s}\left(\mathbb{R}^{d}\right)\right)\right. & \subset \operatorname{ran}\left(S^{(p)}(\lambda) \uparrow H^{s+2(q+1)}\left(\mathbb{R}^{d}\right)\right) \\
& \subset H^{s+2(p+q+1)+1 / 2}(\Sigma)=H^{s+2(l+1)+1 / 2}(\Sigma),
\end{aligned}
$$

which shows (3.10) for $k=l+1$ and hence, by induction, for all $k \in \mathbb{N}_{0}$. Therefore, an application of Lemma 2.1 with $\mathcal{K}=L^{2}(\Sigma)$ and $r=2 k+1 / 2$ yields that

$$
S^{(k)}(\lambda) \in \mathfrak{S}_{\frac{d-1}{2 k+1 / 2}, \infty}, \quad k \in \mathbb{N}_{0}, \lambda \in \mathbb{C} \backslash \mathbb{R}_{+} .
$$

Step 2. Using Krein's formula in (2.13) and (2.5a) we obtain that, for $m \in \mathbb{N}$ and $\lambda \in \mathbb{C} \backslash \mathbb{R}_{+}$,

$$
\begin{aligned}
\widehat{D}_{m}(\lambda) & =\frac{1}{(m-1) !} \cdot \frac{\mathrm{d}^{m-1}}{\mathrm{~d} \lambda^{m-1}}\left(\widehat{D}_{1}(\lambda)\right)=\frac{1}{(m-1) !} \cdot \frac{\mathrm{d}^{m-1}}{\mathrm{~d} \lambda^{m-1}}(\widehat{\gamma}(\lambda) S(\lambda)) \\
& =\frac{1}{(m-1) !} \sum_{\substack{p+q=m-1 \\
p, q \geq 0}}\left(\begin{array}{c}
m-1 \\
p
\end{array}\right) \widehat{\gamma}^{(p)}(\lambda) S^{(q)}(\lambda) .
\end{aligned}
$$

By Lemma $3.2(\mathrm{i}),(3.12)$ and (2.2) we have

$$
\widehat{\gamma}^{(p)}(\lambda) S^{(q)}(\lambda) \in \mathfrak{S}_{\frac{d-1}{2 p+3 / 2}, \infty} \cdot \mathfrak{S}_{\frac{d-1}{2 q+1 / 2}, \infty}=\mathfrak{S}_{\frac{d-1}{2(p+q)+2}, \infty}=\mathfrak{S}_{\frac{d-1}{2 m}, \infty}
$$

for $p, q$ with $p+q=m-1$.

Step 3. If $m>\frac{d-1}{2}$, then $\frac{d-1}{2 m}<1$ and, by (3.14), each term in the sum in (3.13) is a trace class operator and, by a similar argument, also $S^{(q)}(\lambda) \widehat{\gamma}^{(p)}(\lambda)$. Hence, the resolvent power difference $\widehat{D}_{m}(\lambda)$ is a trace class operator, and we can apply the trace to (3.13) and use (2.3), (2.4) and (2.8c) to obtain

$$
\begin{aligned}
& (m-1) ! \operatorname{Tr}\left(\widehat{D}_{m}(\lambda)\right)=\operatorname{Tr}\left(\sum_{\substack{p+q=m-1 \\
p, q \geq 0}}\left(\begin{array}{c}
m-1 \\
p
\end{array}\right) \widehat{\gamma}^{(p)}(\lambda) S^{(q)}(\lambda)\right) \\
& =\sum_{\substack{p+q=m-1 \\
p, q \geq 0}}\left(\begin{array}{c}
m-1 \\
p
\end{array}\right) \operatorname{Tr}\left(\widehat{\gamma}^{(p)}(\lambda) S^{(q)}(\lambda)\right) \\
& =\sum_{\substack{p=m-1 \\
p, q \geq 0}}\left(\begin{array}{c}
m-1 \\
p
\end{array}\right) \operatorname{Tr}\left(S^{(q)}(\lambda) \widehat{\gamma}^{(p)}(\lambda)\right) \\
& =\operatorname{Tr}\left(\sum_{\substack{p+q=m-1 \\
p, q \geq 0}}\left(\begin{array}{c}
m-1 \\
p
\end{array}\right) S^{(q)}(\lambda) \widehat{\gamma}^{(p)}(\lambda)\right) \\
& =\operatorname{Tr}\left(\frac{\mathrm{d}^{m-1}}{\mathrm{~d} \lambda^{m-1}}(S(\lambda) \widehat{\gamma}(\lambda))\right)
\end{aligned}
$$




$$
\begin{aligned}
& =\operatorname{Tr}\left(\frac{\mathrm{d}^{m-1}}{\mathrm{~d} \lambda^{m-1}}\left(\widehat{M}(\lambda)^{-1} \widehat{\gamma}(\bar{\lambda})^{*} \widehat{\gamma}(\lambda)\right)\right) \\
& =\operatorname{Tr}\left(\frac{\mathrm{d}^{m-1}}{\mathrm{~d} \lambda^{m-1}}\left(\widehat{M}(\lambda)^{-1} \widehat{M}^{\prime}(\lambda)\right)\right),
\end{aligned}
$$

which finishes the proof.

Proof of Theorem 1.3. (i) The proof of this statement is fully analogous to the proof of Theorem 1.2. One has to replace in the argument $\mathrm{H}_{\text {free }}, \alpha, \mathrm{H}_{\alpha, \Sigma}, \widetilde{M}, \widetilde{\gamma}$, by $\mathrm{K}_{\mathrm{N}}, \omega, \mathrm{K}_{\omega, \Sigma}, \widehat{M}, \widehat{\gamma}$, respectively, Moreover, Krein's resolvent formula is used in (2.14b) instead of Krein's formula in (2.14a) and Lemma 3.2 instead of Lemma 3.1.

(ii) By item (i) of this theorem and by Lemma 3.4, for every $m \in \mathbb{N}$ such that $m>\frac{d-1}{2}$ and every $\lambda \in \rho\left(\mathrm{K}_{\omega, \Sigma}\right)$ both operators $\widehat{D}_{m}(\lambda)$ and $\widehat{D}_{\omega, m}(\lambda)$ belong to the trace class. In view of the identity $\widehat{E}_{\omega, m}(\lambda)=\widehat{D}_{m}(\lambda)+\widehat{D}_{\omega, m}(\lambda)$, we infer that $\widehat{E}_{\omega, m}(\lambda)$ is also in the trace class. Using the formula $(2.3)$ we have

$$
\operatorname{Tr}\left(\widehat{E}_{\omega, m}(\lambda)\right)=\operatorname{Tr}\left(\widehat{D}_{\omega, m}(\lambda)\right)+\operatorname{Tr}\left(\widehat{D}_{m}(\lambda)\right) .
$$

Combining the trace formula in (i) of this theorem and the trace formula in Lemma 3.4 we obtain

$$
\begin{aligned}
\operatorname{Tr}\left(\widehat{E}_{\omega, m}(\lambda)\right) & =\frac{1}{(m-1) !} \operatorname{Tr}\left(\frac{\mathrm{d}^{m-1}}{\mathrm{~d} \lambda^{m-1}}\left((I-\omega \widehat{M}(\lambda))^{-1} \omega \widehat{M}^{\prime}(\lambda)+\widehat{M}(\lambda)^{-1} \widehat{M}^{\prime}(\lambda)\right)\right) \\
& =\frac{1}{(m-1) !} \operatorname{Tr}\left(\frac{\mathrm{d}^{m-1}}{\mathrm{~d} \lambda^{m-1}}\left((I-\omega \widehat{M}(\lambda))^{-1} \widehat{M}(\lambda)^{-1} \widehat{M}^{\prime}(\lambda)\right),\right.
\end{aligned}
$$

which finishes the proof.

\section{References}

[1] J.-P. Antoine, F. Gesztesy and J. Shabani, Exactly solvable models of sphere interactions in quantum mechanics. J. Phys. A 20 (1987), 3687-3712.

[2] W. Arendt and R. Mazzeo, Friedlander's eigenvalue inequalities and the Dirichletto-Neumann semigroup. Commun. Pure Appl. Anal. 11 (2012), 2201-2212.

[3] W. Arendt and A. F. M. ter Elst, The Dirichlet-to-Neumann operator on rough domains. J. Differential Equations 251 (2011), 2100-2124.

[4] W. Arendt and A. F. M. ter Elst, The Dirichlet-to-Neumann operator on exterior domains. Potential Anal. 43 (2015), 313-340.

[5] C. Bardos, J.-C. Guillot and J. Ralston, La rélation de Poisson pour l'équation des ondes dans un ouvert non borne. Application Ã la théorie de la diffusion. Commun. Partial Differ. Equations 7 (1982), 905-958.

[6] J. Behrndt, P. Exner and V. Lotoreichik, Schrödinger operators with $\delta$ - and $\delta^{\prime}$ interactions on Lipschitz surfaces and chromatic numbers of associated partitions. Rev. Math. Phys. 26 (2014), 1450015, 43 pp. 
[7] J. Behrndt, G. Grubb, M. Langer and V. Lotoreichik, Spectral asymptotics for resolvent differences of elliptic operators with $\delta$ and $\delta^{\prime}$-interactions on hypersurfaces. J. Spectr. Theory 5 (2015), 697-729.

[8] J. Behrndt and M. Langer, Boundary value problems for elliptic partial differential operators on bounded domains. J. Funct. Anal. 243 (2007), 536-565.

[9] J. Behrndt and M. Langer, Elliptic operators, Dirichlet-to-Neumann maps and quasi boundary triples. In Operator methods for boundary value problems, London Math. Soc. Lecture Note Series 404, Cambridge Univ. Press, 2012, 121-160.

[10] J. Behrndt, M. Langer and V. Lotoreichik, Schrödinger operators with $\delta$ and $\delta^{\prime}$ potentials supported on hypersurfaces. Ann. Henri Poincaré 14 (2013), 385-423.

[11] J. Behrndt, M. Langer and V. Lotoreichik, Spectral estimates for resolvent differences of self-adjoint elliptic operators. Integral Equations Operator Theory 77 (2013), 1-37.

[12] J. Behrndt, M. Langer and V. Lotoreichik, Trace formulae and singular values of resolvent power differences of self-adjoint elliptic operators. J. Lond. Math. Soc. (2) 88 (2013), 319-337.

[13] J. Behrndt and J. Rohleder, Spectral analysis of selfadjoint elliptic differential operators. Dirichlet-to-Neumann maps, and abstract Weyl functions. Adv. Math. $\mathbf{2 8 5}$ (2015), 1301-1338.

[14] J.F. Brasche, P. Exner, Y.A. Kuperin and P. Šeba, Schrödinger operators with singular interactions. J. Math. Anal. Appl. 184 (1994), 112-139.

[15] J.F. Brasche and A. Teta, Spectral analysis and scattering theory for Schrödinger operators with an interaction supported by a regular curve. In Ideas and methods in quantum and statistical physics (Oslo, 1988), Cambridge Univ. Press, Cambridge, 1992, 197-211.

[16] V.M. Bruk, A certain class of boundary value problems with a spectral parameter in the boundary condition. Mat. Sb. (N.S.) 100(142) (1976), 210-216.

[17] J. Brüning, V. Geyler and K. Pankrashkin, Spectra of self-adjoint extensions and applications to solvable Schrödinger operators. Rev. Math. Phys. 20 (2008), 1-70.

[18] V.S. Buslaev and L. D. Faddeev, Formulas for traces for a singular Sturm-Liouville differential operator. Sov. Math. Dokl. 1 (1960), 451-454.

[19] G. Carron, Determinant relatif et la fonction Xi. Amer. J. Math. 124 (2002), 307352 .

[20] S. Clark, F. Gesztesy, R. Nichols and M. Zinchenko, Boundary data maps and Krein's resolvent formula for Sturm-Liouville operators on a finite interval. Oper. Matrices 8 (2014), 1-71.

[21] V.A. Derkach and M. M. Malamud, Generalized resolvents and the boundary value problems for Hermitian operators with gaps. J. Funct. Anal. 95 (1991), 1-95.

[22] V.A. Derkach and M. M. Malamud, The extension theory of Hermitian operators and the moment problem. J. Math. Sci. 73 (1995), 141-242.

[23] J. Dittrich, P. Exner, C. Kühn and K. Pankrashkin, On eigenvalue asymptotics for strong delta-interactions supported by surfaces with boundaries. Asymptotic Anal., to appear. Preprint: arXiv:1506.06583.

[24] V. Duchêne and N. Raymond, Spectral asymptotics of a broken $\delta$-interaction. $J$. Phys. A 47 (2014), 155203, 19 pp. 
[25] A. F. M. ter Elst and E. M. Ouhabaz, Analysis of the heat kernel of the Dirichlet-toNeumann operator. J. Funct. Anal. 267 (2014), 4066-4109.

[26] P. Exner, Leaky quantum graphs: a review. In Analysis on graphs and its applications, Proc. Sympos. Pure Math. 77, Amer. Math. Soc., Providence, RI, 2008, $523-564$.

[27] P. Exner, E. M. Harrell and M. Loss, Inequalities for means of chords, with application to isoperimetric problems. Lett. Math. Phys. 75 (2006), 225-233.

[28] P. Exner and T. Ichinose, Geometrically induced spectrum in curved leaky wires. $J$. Phys. A 34 (2001), 1439-1450.

[29] P. Exner and M. Jex, Spectral asymptotics of a strong $\delta^{\prime}$ interaction on a planar loop. J. Phys. A 46 (2013), 345201, 12 pp.

[30] P. Exner and H. Kovařík, Quantum waveguides. Theoretical and Mathematical Physics, Springer, Cham, 2015.

[31] L. Friedlander, Absolute continuity of the spectra of periodic waveguides. In Waves in periodic and random media (South Hadley, MA, 2002), Contemp. Math. 339, Amer. Math. Soc., Providence, RI, 2003, 37-42.

[32] F. Gesztesy and M. Zinchenko, Symmetrized perturbation determinants and applications to boundary data maps and Krein-type resolvent formulas. Proc. Lond. Math. Soc. (3) 104 (2012), 577-612.

[33] I. C. Gohberg and M. G. Krĕn, Introduction to the theory of linear nonselfadjoint operators. Translations of Mathematical Monographs 18, Amer. Math. Soc., Providence, RI, 1969.

[34] G. Grubb, Remarks on trace estimates for exterior boundary problems. Commun. Partial Differ. Equations 9 (1984), 231-270.

[35] M. Jex and V. Lotoreichik, On absence of bound states for weakly attractive $\delta^{\prime}$ interactions supported on non-closed curves in $\mathbb{R}^{2}$. J. Math. Phys., to appear. Preprint: arXiv:1508.04577.

[36] R. Jost and A. Pais, On the scattering of a particle by a static potential. Physical Rev. (2) 82 (1951), 840-851.

[37] T. Kato, Perturbation theory for linear operators. Reprint of the 1980 edition. Classics in Mathematics, Springer-Verlag, Berlin, 1995.

[38] R. Killip and B. Simon, Sum rules for Jacobi matrices and their applications to spectral theory. Ann. Math. (2) 158 (2003), 253-321.

[39] R. Killip and B. Simon, Sum rules and spectral measures of Schrödinger operators with $L^{2}$-potentials. Ann. Math. (2) 170 (2009), 739-782.

[40] A. N. Kočubeř, Extensions of symmetric operators and of symmetric binary relations. Mat. Zametki 17 (1975), 41-48.

[41] S. Kondej and V. Lotoreichik, Weakly coupled bound state of 2-D Schrödinger operator with potential-measure. J. Math. Anal. Appl. 420 (2014), 1416-1438.

[42] P. Lax and R. Phillips, Decaying modes for the wave equation in the exterior of an obstacle. Commun. Pure Appl. Math. 22 (1969), 737-787.

[43] J.-L. Lions and E. Magenes, Non-homogeneous boundary value problems and applications. Vol. I. Die Grundlehren der mathematischen Wissenschaften 181, SpringerVerlag, New York-Heidelberg, 1972. 
[44] V. Lotoreichik and T. Ourmières-Bonafos, On the bound states of Schrödinger operators with $\delta$-interactions on conical surfaces. Preprint: arXiv:1510.05623.

[45] W. McLean, Strongly elliptic systems and boundary integral equations. Cambridge University Press, Cambridge, 2000.

[46] R. Melrose, Weyl asymptotics for the phase in obstacle scattering. Commun. Partial Differ. Equations 13 (1988), 1431-1439.

[47] B. Simon, Trace ideals and their applications. Second edition. Mathematical Surveys and Monographs 120, Amer. Math. Soc. Providence, RI, 2005.

[48] O. Steinbach, Numerical approximation methods for elliptic boundary value problems. Finite and boundary elements. Springer, New York, 2008.

Jussi Behrndt, Institut für Numerische Mathematik, Technische Universität Graz, Steyrergasse 30, 8010 Graz, Austria

E-mail: behrndt@tugraz.at

Matthias Langer, Department of Mathematics and Statistics, University of Strathclyde, 26 Richmond Street, Glasgow G1 1XH, United Kingdom

E-mail: m.langer@strath.ac.uk

Vladimir Lotoreichik, Department of Theoretical Physics, Nuclear Physics Institute, Academy of Sciences, 250 68, Řež near Prague, Czech Republic

E-mail: lotoreichik@ujf.cas.cz 